III. MASS TRANSFER AND MASS LOSS - THEORY 


\title{
CLOSE BINARIES BEFORE AND AFTER MASS EXCHANGE: A COMPARISON OF OBSERVATIONS THROUGH THEORETICAL COMPUTATIONS(1)
}

\author{
J.P. De Grève(2) and D. Vanbeveren \\ Astrophysical Institute, Vrije Universiteit Brussel, \\ Brussels, Belgium.
}

From a search through the 1 iterature 174 close binaries with completely known absolute dimensions are sampled. Distinction is made between systems before and after mass exchange, giving resp. 100 and 40 systems (a third group contains the systems not definitely belonging to these two). Mass, period and mass ratio distributions and relations of the group of "unevolved" binaries (i.e. prior to mass exchange) are transformed into corresponding distributions and relations of evolved binaries. The transformations are based upon the $M_{1 f}=g\left(M_{1 i}\right)$ relation derived from an extended set of published theoretical computations on the evolution of close binaries. Final masses resulting from the same initial mass are averaged. Equations are derived for the cases $A$ (for a 11 masses), B1 $\left(M_{1 i} / M_{0} \leq 2.8\right), B 2\left(2.8<M_{1 i} / M_{0} \leq 9\right)$ and $B 3\left(M_{1 j} / M_{0}>9\right)$. For the changes of the period due to angular momentum loss the formalism of Vanbeveren et al. (1979) was adopted. The following characteristics of the system after mass exchange are computed: $M_{1 f}, M_{2} f\left(\right.$ and $q_{f}$ ), $P_{f}$. Three different modes were applied for the mass loss from the system:

a) conservative case (mass and angular momentum of the system remain constant), called $C$.

b) non conservative case with $50 \%$ of the transferred mass leaving the system with a small or a large angular momentum loss (resp. called NC51 and NC53).

c) non conservative case with $100 \%$ of the transferred mass leaving the system with a small or a large angular momentum loss (resp. called NC101 and NC103).

The distributions of the parameters $M_{1}, q^{-1}$ and $P$ of the transformed unevolved systems are compared to the corresponding distributions of observed evolved systems. The most important features of the comparison are given in tables 1 , 2 and 3 . From this comparison the following conclusions are derived:

1. The mass distribution of the unevolved systems gives a fair representation of reality in the range $1.6 M_{0}-15 M_{0}$, 103

M. J. Plavec, D. M. Popper and R. K. Ulrich (eds.), Close Binary Stars: Observations and Interpretation, $103-107$. Copyright $\odot 1980$ by the IAU. 
Table 1. Comparison of the mass distribution of evolved primaries and transformed ones in the range $-0.4<$ $\log M_{1}<0.6$. The transformed systems have been normalized to the total number of evolved systems in that mass range.

\begin{tabular}{|c|c|c|c|c|c|}
\hline $\log M_{1}-b i n$ & $-0.4-0.2$ & -0.20 .0 & 0.00 .2 & 0.20 .4 & 0.40 .6 \\
\hline $\begin{array}{l}\text { nr of evolved } \\
\text { systems }\end{array}$ & 12 & 10 & 5 & 3 & 3 \\
\hline $\begin{array}{l}n r \text { of } \\
\text { transformed } \\
\text { systems }\end{array}$ & 13 & 11 & 5 & 2 & 2 \\
\hline
\end{tabular}

Table 2. Comparison of characteristics of the $q^{-1}$ distributions of set II', with the distribution of observed evolved systems (set II).

\begin{tabular}{|lccccc|}
\hline \multicolumn{1}{|c}{ Group } & $\begin{array}{c}\text { Maximum } \\
\mathrm{q}_{1}<\mathrm{q}^{-1} \leq \mathrm{q}_{2}\end{array}$ & of $\max$. & $\begin{array}{c}\text { in } \\
0.1<\mathrm{q}^{-1} \leq 0.3\end{array}$ & with $\mathrm{q}^{-1}>0.6$ \\
\hline Observations & $0.2-0.3$ & 30 & 57 & 0 \\
Conservative & $0.1-0.2$ & 43 & 50 & 3 \\
NC50 & $0.1-0.2$ & 55 & 74 & 3 \\
NC100 & $0.2-0.3$ & 29 & 57 & 11 \\
\hline
\end{tabular}

Table 3. The same as Table 2, but for the period distribution.

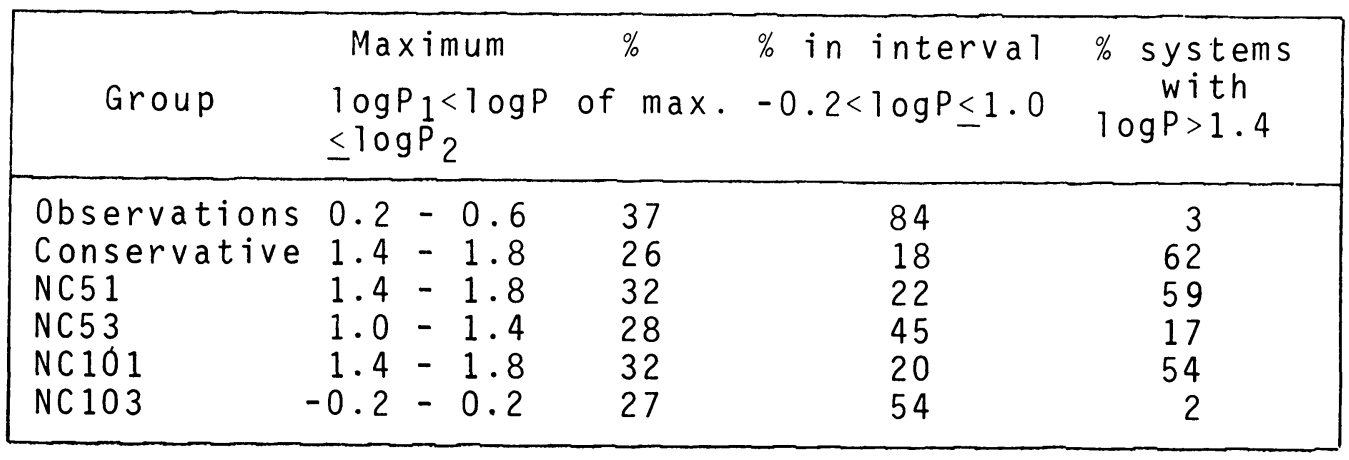

the low mass stars being slightly underabundant; for the evolved system good correspondence is found in the range $0.4 \mathrm{M}_{0}-2.5 \mathrm{M}_{0}$.

2. The theoretical formalism of $M_{1}$ depending on $M_{1}$ results in a striking correspondence between set I I and set II' (= the transformed set I).

3. The best quantitative correspondence for the $q^{-1}$ 
distribution is found with mode NC100; the appearance of systems with mass ratios $q^{-1}>0.6$ for that mode may indicate that the external mass loss is influenced by the parameters $M_{1}, q, P$.

4. The best correspondence in the period distributions is obtained when a large amount of angular momentum is removed from the system; a still better agreement results if one assumes that not all, byt say $80 \%$ of the transferred mass is leaving the system. This also causes a strong decrease of the excess of transformed short period systems, appearing with mode NC103.

5. Only a very small fraction of the evolved systems can be transformed into unevolved by using the conservative mode. The average value of the maximum fraction of mass transferred to the secondary (in order to obtain a nonnegative initial mass of the secondary) is $\sim 0.6$.

The different distributions were analysed against selection effects. It was found that the selection effects in the distributions of $i_{1}, q$ and $P$ do not alter the conclusions at all.

\section{REFERENCE}

Vanbeveren, D., De Grève, J.P., van Dessel, E.L., de Loore, C.: 1979, Astron.Astrophys. 73, 19.

\section{Notes}

(1) For the details of this investigation we refer to De Grève, J.P. and Vanbeveren, D., Astrophys. Space Sci. (submitted).

(2) Research Fellow, F.K.F.0., Belgium.

COMMENTS FOLLOWING DE GRÈVE AND VANBEVEREN

Meyer-Hofmeister: For a comparison we need complete samples of observed and computed evolved binaries. Initial distributions of binary masses and separations are needed to determine the distribution of evolved binaries. How did you determine the sample of evolved (computed) binaries?

De Grève: Computed evolved binaries (called "transformed") are constructed by transforming each observed unevolved system in the following way. The mass of the loser is transformed into a remnant mass $q\left(M_{i}\right)$. The mass of the gainer is determined by the assumption on the mass ${ }^{i}$ leaving the system $(0,50$ or $100 \%)$, resulting in a value $\mathrm{q}_{\mathrm{f}}^{-1}$. The final period is computed with the formalism quoted, adopting assumptions on the angular momentum loss. Thus several sets of 
transformed systems are constructed. These sets are compared to the set of observed evolved binaries. The influence of selection effects is discussed in the comparison, but herefore I refer to the extended mass paper. For example for the mass distributions the ranges where the distributions are close to reality are determined as well as the proper fractions of systems, taking into account evolutionary aspects, such as different lifetimes for different masses.

Smak: I am still under an impression that, because of the severe selection effects affecting your main sequence input sample and the semi-detached sample, no meaningful comparison can be made.

De Greve: It is so that for the mass distributions of unevolved and evolved systems selection effects were examined by constructing different mass distributions within well defined space volumes from the catalogue of Batten and supplements to it. The comparison shown in table 1 refers only to the mass range where the mass distributions of both set I and II are close to the volume distributions. For the period and mass ratio distribution also arguments are given (in the extended paper) to show that the selection effects in the distributions do not alter the comparison made in tables 2 and 3 .

Sugimoto: Since the physical situations are very complicated and many parameters are involved, it is better to ask the following questions. How much uncertainty, do you think, is involved in your conclusion of 80 percent mass loss? Do you think it possible to obtain any quantitative result concerning the amount of the angular momentum loss from such considerations?

De Grève: I do agree that many parameters are involved and that precise quantitative results on mass and angular momentum loss vary from system to system. The 80 percent quoted in my talk is to be interpreted as an average result. It is difficult to say something about the uncertainty but I could mention two aspects that give at least an idea:

1 - If one assumes that less than 50 percent of the transferred mass leaves the system, the resulting $\mathrm{q}^{-1}$ and $\mathrm{P}$-distributions differ rather strongly from the observed.

2 - The fact that the average maximum fraction of the mass accreted by the secondary, as derived from the determination of initial parameters of evolved systems, is 0.6. I think that a detailed analysis of these minima for an extensive set of evolved systems can give more information.

Plavec: Typical detached systems have periods of a few days, and the relative dimensions of the components are so large that when the more massive one overflows, the stream hits the other star. It is therefore rather surprising to hear that almost all the material should leave the system. This would imply that these stars probably are not the progenitors of Algols, and that the real progenitors are wider systems that we probably do not observe. 
De Grève: The fact that most of the material should leave the system is not so surprising. Theoretical computations have shown that either the stream hits the gainer or falls on it through a ring structure; the gainer swells up rapidly and fills its own critical lobe, forcing the material to leave through $\mathrm{L}_{1}$. Only in the last stage of mass transfer when the loser has already lost the largest fraction of the material, the gainer reacts less violently to accretion. Whether or not the progenitors of Algols should be searched among more wide systems is not so easy to determine. From the comparison of the distribution values for the parameters determining the mass and angular momentum loss result in a ratio of initial to final period near or larger than unity, indicating that the Algol-progenitors may still result from systems with periods of a few days.

de Loore: I want to make a comment concerning the value of the mass and angular momentum losses. It should be kept in mind that these results are global results, and the conclusion is that mass and angular momentum losses are very large. The values of $2100 \%$ quoted within this paper should be interpreted in this sense.

Most probably these losses are different from system to system; it should not be concluded that none of the secondaries can accrete a part of the expelled matter. 